\title{
Rib resection using a Gigli saw under thoracoscopic guidance
}

\author{
Ilhan Ocakcioglu ${ }^{1}$, Fuat Sayir ${ }^{2}$ \\ ${ }^{1}$ Department of Thoracic Surgery, Pendik Training and Research Hospital, Marmara University, Istanbul, Turkey \\ ${ }^{2}$ Department of Thoracic Surgery, Medicine Faculty, Yüzüncü Yıl University, Van, Turkey
}

Videosurgery Miniinv 2019; 14 (1): 126-132

DOI: https://doi.org/10.5114/wiitm.2018.77266

\begin{abstract}
Introduction: Despite the advantages and expanded indications of video-assisted thoracoscopic surgery, the open surgical approach is commonly preferred for the surgical treatment of rib lesions. Such an approach could lead to disadvantageous results such as increased postoperative pain and prolonged hospital stay. Despite all these handicaps, thoracoscopic resection of isolated rib resection has been reported in a small number of publications.

Aim: To compare the clinical outcomes of patients with isolated benign rib pathologies treated with either minimally invasive or open surgery.

Material and methods: The medical records of 22 patients undergoing surgery for isolated benign rib pathologies between 2013 and 2017 were reviewed. Variables statistically compared between the two groups were age, gender, symptoms, lesion size, duration of the surgery, amount of intraoperative bleeding, conversion to open surgery, volume and duration of the drainage, postoperative complications, length of hospital stay, pathological diagnosis, follow-up period, recurrence, duration of narcotic analgesic usage and pain according to visual analog scale evaluation. Results: The thoracoscopic approach was superior to conventional surgery in terms of drainage volume, time to drain removal, morbidity, hospital stay, narcotic analgesic treatment duration and postoperative pain scores. All thoracoscopic procedures were concluded successfully, and conversion to open surgery was not required. During the mean 14-month follow-up period, no recurrence was encountered in either group.

Conclusions: This technique is a safe option for isolated benign lesions of the ribs. It is more effective in patient recovery in the postoperative period and in the management of surgical pain.
\end{abstract}

Key words: resection, thoracoscopy, rib, Gigli saw.

\section{Introduction}

The prevalence of rib tumors ranges from 3 to $8 \%$ depending on the series [1, 2]. 50-80\% of rib pathologies are malignant. The most common malignant rib tumors are metastases and myeloma. Osteochondroma accounts for $50 \%$ of all benign tumors [3]. These lesions are often discovered incidentally.

Increasing evidence suggests that video-assisted thoracoscopic surgery (VATS) is an alternative method for conventional thoracotomy in thoracic surgery. Nowadays, thoracoscopy, either single or multipor- tal, is administered in a changing spectrum from pneumothorax to anatomic resections of the lung $[4,5]$. Despite all these applications, thoracoscopic resection of the chest wall and isolated rib resection have been reported in several publications. The open surgical approach is commonly preferred for the surgical treatment of rib pathologies. The VATS rib resection is considered a difficult procedure by majority of surgeons. In contrast, isolated rib lesions such as osteochondroma, enchondroma, or other benign tumors can be easily treated thoracoscopically. 


\section{Aim}

In this study, we evaluated the feasibility of safe thoracoscopic resection of a rib segment using a Gigli saw, instead of conventional surgery. We attempt to compare the clinical outcomes of patients with isolated rib pathologies treated with either minimally invasive or open surgery.

\section{Material and methods}

Between 2013 and 2017 patients who underwent surgery for rib pathologies were retrospectively reviewed. All patients were evaluated clinically and radiologically with chest $\mathrm{X}$-ray, computed tomography and bone scintigraphy (Photos $1 \mathrm{~A}-\mathrm{C}$ ). The lesions greater than $5 \mathrm{~cm}$ in diameter underwent preoperative needle aspiration or incisional biopsy.

Multiple rib involvement, malign lesions, metastatic lesions, local invasion of the lung and pleural tumor infiltrating the chest wall were not included in the study. Patients having solitary benign rib pathology were selected for the study. Reconstruction of the chest wall was not required in defects less than $5 \mathrm{~cm}$ in diameter and posterior defects covered by the scapula.
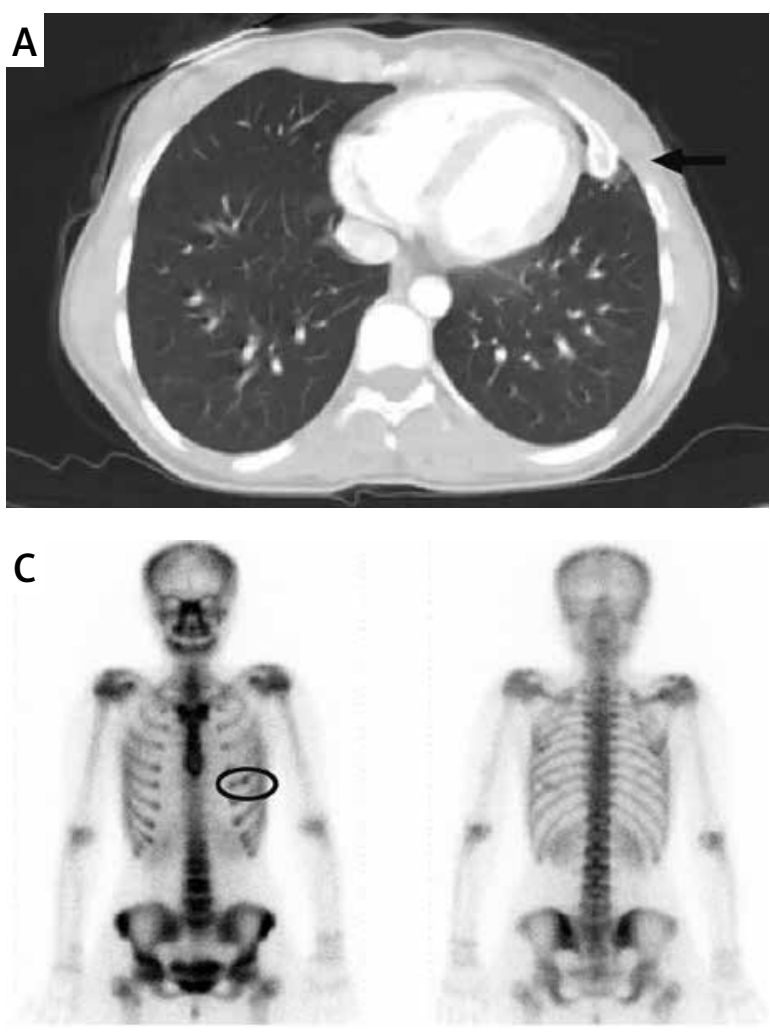

Variables statistically compared between the two groups were age, gender, symptoms, lesion size, duration of the surgery, amount of intraoperative bleeding, conversion to open surgery, volume and duration of drainage, postoperative complications, length of hospital stay, pathological diagnosis, follow-up period, recurrence, duration of narcotic analgesic (NA) usage and pain according to visual analog scale (VAS) evaluation.

Each of these two approaches was performed by the same surgeon throughout the study, using the approach they are most experienced in (VATS by IO, open surgery by FS). All patients were informed in detail about the surgical procedure, risks, and complications, and written consent was waived preoperatively.

\section{Surgical technique}

\section{Open surgery}

The open surgical approach was performed with two different surgical procedures. Thoracotomy was preferred in large diameter lesions or posterior lesions covered by the scapula. Second option for open surgery was preferred in smaller diameter lesions.

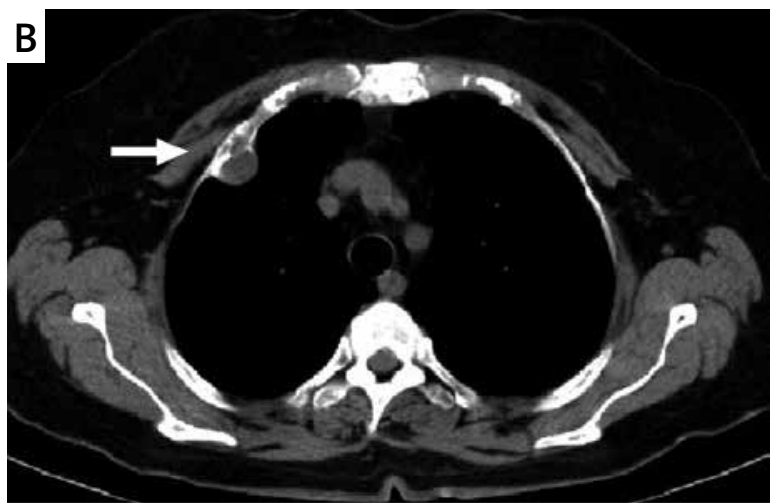

Photo 1. A - Axial plain CT shows bony outgrowth from the posterior cortex of left $5^{\text {th }}$ rib. B - CT shows $1.8 \times 1.7 \mathrm{~cm}$ size mass in the right second rib. $\mathbf{C}-$ Bone scintigraphy shows increased activity in the left $5^{\text {th }}$ rib 
In the first, the operation was performed via a standard posterolateral thoracotomy incision following double lumen intubation. The diseased rib segment was resected leaving a safe surgical margin of at least $2.5 \mathrm{~cm}$ proximal and distal to the tumor. The upper and lower muscle and pleura tissue adjacent to the tumor were included in the resection.

For the other, open procedure; the patient was placed in the lateral decubitus position after the single lumen intubation. According to the possible location of the lesion in computed tomography, muscle and other soft tissue structures were separated by a soft tissue retractor following the skin incision. The resection of the rib segment was performed with similar surgical principles. A single chest tube was placed into the thorax at the end of both procedures.

Defects less than $5 \mathrm{~cm}$ and posterior defects covered by the scapula typically did not require reconstruction. Skeletal stabilization in defects larger than $5 \mathrm{~cm}$ was achieved with prolene mesh reconstruction.

\section{Video-assisted thoracoscopic surgery}

The patient was placed in the lateral decubitus position and double lumen intubation was performed
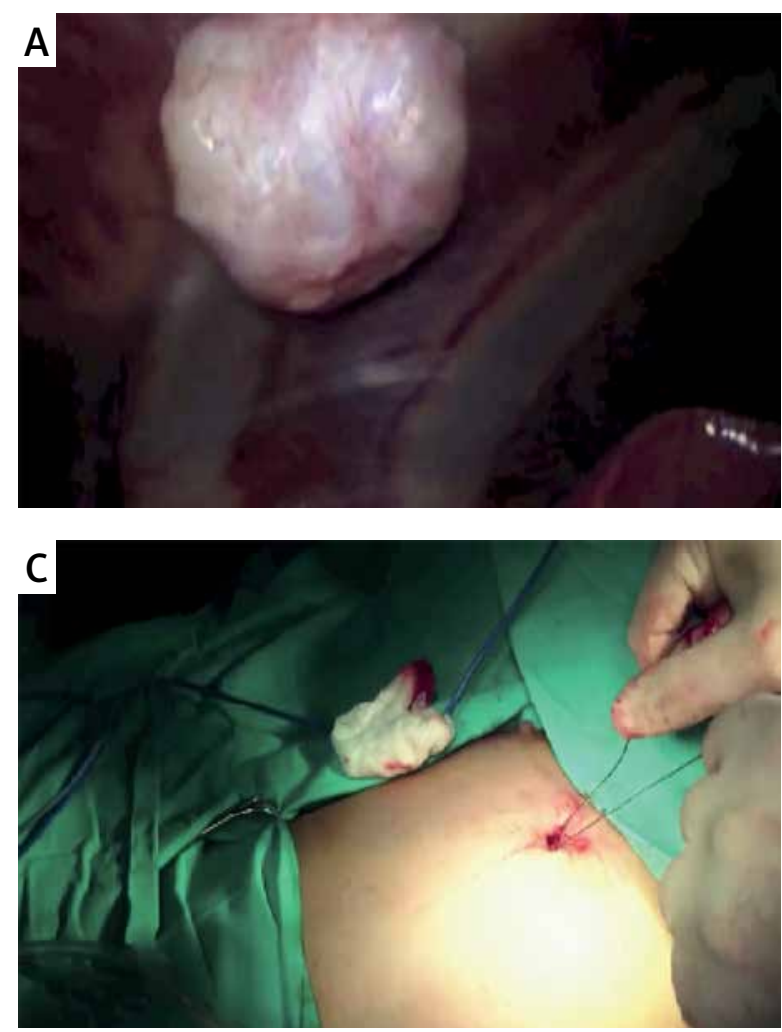

to achieve unilateral ventilation. The first incision was a 2-cm port incision for the 30-degree thoracoscope (Karl Storz endoscope; Karl Storz, Tuttlingen, Germany). The port setup depended on the localization of the tumor. It was placed at the level of the sixth or seventh intercostal space. The borders of the rib lesion were marked with needles, and two additional ports were placed $2.5 \mathrm{~cm}$ anterior and posterior to the lesion under thoracoscopic control. The parietal pleura inferior to the rib was opened with electrocautery. Periosteal tissues at the proximal and distal ends of the rib were dissected. The intercostal vessels were coagulated and cut with electrocautery. The lower margin of the rib was looped with a Gigli saw, and the rib was transected (Photos $2 \mathrm{~A}-\mathrm{C}$ ). The upper margin of the rib was transected in the same manner. A segment of the rib was resected, and a bag was inserted into the thoracic cavity through the port for removal of the rib segment (Photo 3). The operative port incisions were sutured and one chest tube was inserted via the camera port wound.

\section{Statistical analysis}

Postoperative data on clinical outcomes were collected and analyzed retrospectively. Descriptive

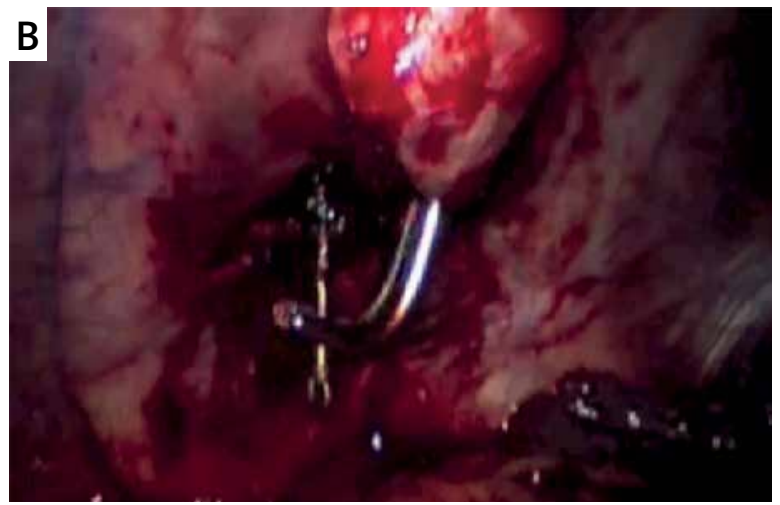

Photo 2. A-Lesion (osteochondroma). B, C-The lower and the upper margin of the rib are looped with a Gigli saw, and the rib is partially resected 
statistics for continuous variables were reported as means and standard deviation (SD) with 95\% confidence interval $(\mathrm{Cl})$ and were compared using the Mann-Whitney $U$ test. The $\chi^{2}$ test was used to determine the relationship between groups and categorical variables. In this study, the maximum type I error was 0.05 , and the level of significance was accepted as $p<0.05$. All analyses were performed using SPSS software (SPSS Inc., Chicago, IL, USA).

\section{Results}

Between 2013 and 2017, a total of 22 patients (12 males and 10 females) underwent surgery for rib pathologies. The age of the patients varied from 9 to 60 years (mean: 28.5 years). Symptoms at the time of presentation were chest pain $(40.9 \%)$ and swelling $(18.1 \%)$. Ten $(45.5 \%)$ patients were asymptomatic and the lesion was accidentally found during routine chest radiography.

Of the 22 patients with benign rib pathologies, 8 (36.3\%) patients had enchondroma; 7 (31.8\%) patients osteochondroma; 3 (13.6\%) patients fibrous dysplasia; 1 (4.5\%) patient xanthoma; 1 (4.5\%) patient aneurysmal bone cyst; 1 (4.5\%) patient fibroma and 1 (4.5\%) patient cavernous hemangioma.

The lesion dimensions varied from $1.8 \times 1.7$ to $9 \times 4.5 \mathrm{~cm}$. The mean diameter of the lesions was not significantly different between the two groups (open surgery: $4.43 \pm 2.32 \mathrm{~cm}$; VATS: $2.85 \pm 0.81 \mathrm{~cm}$; $p=0.06$ ).

The number of patients who underwent open surgery was 13 (59\%), while 9 (40.9\%) were managed by VATS. Thoracotomy was preferred in 3 of 13 patients who underwent an open surgery procedure due to the fact that lesion size was large in 2 patients $(9 \times 4.5 \mathrm{~cm}$ and $8 \times 3.5 \mathrm{~cm})$ and the lesion was located under the scapula in 1 patient. Chest wall reconstruction was achieved via prolene mesh in 2 thoracotomy patients with a defect over $5 \mathrm{~cm}$.

All thoracoscopic procedures were concluded successfully, and conversion to open surgery was not required. Of the 22 resections, 14 were on the right side. The mean duration of the procedure was not significantly different between the two groups (open surgery: $62.3 \pm 24.9$ min; VATS: $53.8 \pm 20.4 \mathrm{~min} ; p=$ $0.35)$. No perioperative complications were observed in any case. Patient demographics and perioperative outcomes are shown in Table I.

The drainage volume and time to chest tuberemoval were significantly shorter in the VATS group com-

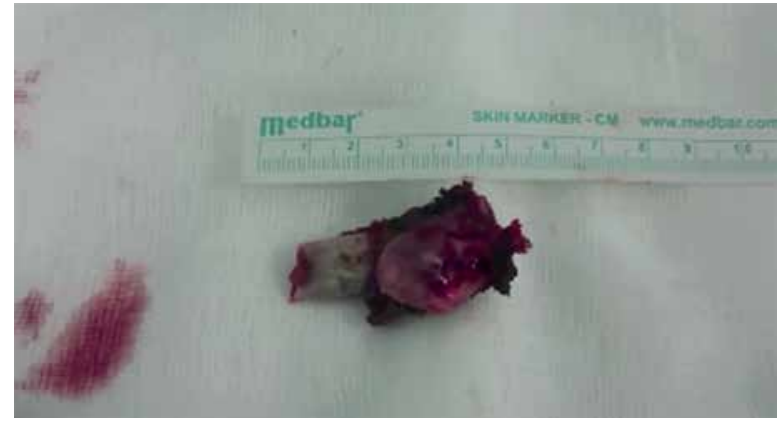

Photo 3. Specimen

pared to the open surgery patients $(86.6 \pm 52.07 \mathrm{ml}$ vs. $157.6 \pm 81.55 \mathrm{ml}$ and $1.78 \pm 0.83$ days vs. 2.91 \pm 1.18 days, respectively; $p=0.04$ and $p=0.03$ ). The total hospital stay, including surgery and postoperative stay, was significantly shorter in the VATS group compared to the open surgery group (2.67 \pm 0.70 days vs. $4.15 \pm 1.51$ days, respectively; $p=0.013$ ).

The postoperative analgesic requirement was significantly lower in the VATS group than in the open surgery group $(1.67 \pm 0.70$ days vs. $3.38 \pm 1.44$ days, respectively; $p=0.04)$. VAS pain score at the postoperative $24^{\text {th }} \mathrm{h}$ was $3.11 \pm 0.78$ in the VATS group, which was significantly lower than in the open surgery group (4.54 \pm 1.12 and $p=0.04$ ). Postoperative outcomes of the two procedures are shown in Table II.

No postoperative mortality was seen in either group. In the VATS group, none of the patients suffered from postoperative complications. Three patients experienced complications postoperatively in the open surgery group; 2 cases of wound infection and 1 case of atelectasis. Fiberoptic bronchoscopy was performed in a patient with atelectasis and a large mucus plug obscuring the right upper lobe bronchus was removed. Follow-up chest radiography demonstrated resolution of the atelectasis. The patients with wound infection were successfully treated with antibiotic therapy. The postoperative complications were significantly lower in the VATS group than in the open surgery group ( $0 \%$ vs. $23.1 \%$ and $p<0.05$ ).

The histopathologic diagnosis of 2 patients having a lesion greater than $5 \mathrm{~cm}$ in diameter was diagnosed by incisional biopsy and fine-needle aspiration. Other patients were diagnosed definitively histopathologically in the postoperative period. All resection margins were free from tumor cells. During the mean 14-month follow-up period, no recurrence was encountered in either group. 
Table I. Patient demographics and perioperative comparison of groups

\begin{tabular}{|c|c|c|c|}
\hline Parameter & VATS & Open surgery & $P$-value \\
\hline Age [years] & $35.11 \pm 15.94$ & $24.08 \pm 10.10$ & 0.06 \\
\hline Gender: & & & 0.93 \\
\hline Male & $5(55.6 \%)$ & $7(53.8 \%)$ & \\
\hline Female & $4(44.4 \%)$ & $6(46.2 \%)$ & \\
\hline Lesion size $[\mathrm{cm}]$ & $2.85 \pm 0.81$ & $4.43 \pm 2.32$ & 0.06 \\
\hline Side: & & & 0.51 \\
\hline Right & $5(55.6 \%)$ & $9(69.2 \%)$ & \\
\hline Left & $4(44.4 \%)$ & $4(30.8 \%)$ & \\
\hline Operation time [min] & $53.8 \pm 20.4$ & $62.3 \pm 24.9$ & 0.35 \\
\hline Intraoperative blood loss [ml] & Minimal & Minimal & \\
\hline Perioperative complication & $0(0 \%)$ & $0(0 \%)$ & \\
\hline
\end{tabular}

Table II. Postoperative evaluation of groups

\begin{tabular}{|c|c|c|c|c|}
\hline \multicolumn{2}{|l|}{ Parameter } & $\begin{array}{c}\text { VATS } \\
\text { Mean } \pm \text { SD }\end{array}$ & $\begin{array}{l}\text { Open surgery } \\
\text { Mean } \pm \text { SD }\end{array}$ & $P$-value \\
\hline \multicolumn{2}{|c|}{ Drainage volume [ml] } & $86.6 \pm 52.07$ & $157.6 \pm 81.55$ & 0.04 \\
\hline \multicolumn{2}{|c|}{ Time to drain removal [days] } & $1.78 \pm 0.83$ & $2.91 \pm 1.18$ & 0.03 \\
\hline \multicolumn{2}{|c|}{ Time to discharge [days] } & $2.67 \pm 0.70$ & $4.15 \pm 1.51$ & 0.01 \\
\hline \multicolumn{2}{|c|}{ NA treatment duration [days] } & $1.67 \pm 0.70$ & $3.38 \pm 1.44$ & 0.04 \\
\hline \multicolumn{2}{|c|}{ Pain VAS scores (24 h) } & $3.11 \pm 0.78$ & $4.54 \pm 1.12$ & 0.04 \\
\hline \multicolumn{2}{|c|}{ Follow-up period [month] } & $14.11 \pm 7.42$ & $14.50 \pm 8.45$ & 0.86 \\
\hline \multicolumn{2}{|l|}{ Parameter } & $n(\%)$ & $n(\%)$ & $P$-value \\
\hline \multirow[t]{2}{*}{ Complications } & None & $9(100 \%)$ & $10(76.9 \%)$ & $<0.05$ \\
\hline & Present & $0(0 \%)$ & $3(23.1 \%)$ & \\
\hline \multicolumn{2}{|l|}{ Recurrence } & $0(0 \%)$ & $0(0 \%)$ & \\
\hline
\end{tabular}

NA - narcotic analgesics, SD - standard deviation, VAS - visual analogue scale, VATS - video-assisted thoracoscopic surgery.

\section{Discussion}

Rib tumors are rare, with an incidence of less than $1 \%$ in the population $[4,5]$. Most rib lesions are malignant tumors, with metastatic involvement or direct invasions from adjacent malignancies such as lung cancer, mesothelioma, breast cancer and mediastinal tumor being the commonest. The most common benign tumors of the chest wall include osteochondroma, fibrous dysplasia, chondroma, and desmoids tumor [6], with many other rarer tumors reported in the literature. For benign rib tumors, surgery must consist of wide resection of the involved ribs with $2-3 \mathrm{~cm}$ free margins to be considered as sufficient.

In the majority of patients, radiographic features alone are insufficient to make a complete diagnosis, and therefore pathologic evaluation is required. Fine-needle aspiration, incisional biopsy, and excisional biopsy are all suitable modalities to obtain a tissue diagnosis. Typically, lesions less than $5 \mathrm{~cm}$ 
undergo excisional biopsy, and lesions greater than $5 \mathrm{~cm}$ may undergo either needle aspiration or incisional biopsy [7]. In our study, the pathologic diagnosis in 2 patients having a lesion greater than $5 \mathrm{~cm}$ diameter was made by incisional biopsy and fine-needle aspiration.

Since the late 1990s, VATS technique has been applied in the treatment of many other types of esophageal, mediastinal and pulmonary diseases $[8,9]$. The role of VATS has expanded substantially following the proof of causing less pain, shorter duration of hospitalization, and esthetically advantageous results [10]. Despite the expanding indications for and advantages of VATS in thoracic surgery, reports on the use of minimally invasive techniques to accomplish isolated rib or chest wall resection are limited. Today, open surgery is widely used for resection of the rib and chest wall.

At first, a few reports were published about techniques using specialized equipment such as the drill [11]. In the following years, Nakagiri et al. [12] first described the thoracoscopic resection of ribs with the help of a Gigli saw that is used for various bone resections. Then Rocco et al. [13] used the Gigli saw for rib resection and additionally performed reconstruction with a titanium plate in order to prevent lung herniation.

This technique uses a commonly available Gigli saw. It is a flexible wire saw used by surgeons for bone cutting. The wire is attached to two Gigli saw handles and moved back and forth about $180^{\circ}$ around the bone.

Although we prefer the Gigli saw for cutting the rib in our study, other techniques can be used. For instance, Ohtsuka et al. reported a thoracoscopic first rib resection for treatment of thoracic outlet syndrome, employing an endoscopic drill [11]. Cameron presented a video using a high-speed burr to divide ribs in chest wall resections [14]. Demmy et al. reported a thoracoscopic en bloc chest wall resection using an endoscopic rib cutter [15].

In our study, VATS was superior to conventional surgery in terms of drainage volume, time to drain removal, morbidity, hospital stay, NA treatment duration and postoperative pain scores. When a conventional approach is used to perform a rib resection, the skin incision must be longer than the rib to be resected. As a result, a conventional rib resection leaves a relatively large and esthetically unfavorable scar. Unlike the traditional approach, VATS allows re- section of ribs via a smaller incision without damaging muscle tissue. A $2 \mathrm{~cm}$ skin incision for the thoracoscope and two $0.5 \mathrm{~cm}$ extra ports where the Gigli saw is used are enough for this procedure. This leads to reductions in the intensity and the duration of postoperative pain and it allows patients to return to full activity more quickly $[10,16]$. In confirmation of the other studies, postoperative $24^{\text {th }}$ hour VAS pain scores were significantly better in the VATS group than the other group $(p=0.04)$. Likewise, the duration of NA treatment was shorter in the VATS group (1.67 \pm 0.70 days vs. $3.38 \pm 1.44$ days). The larger incision and damage of muscle and intercostal nerve tissue are the main reasons for pain in open surgery.

In addition, VATS is an advantageous method over traditional surgical practices, due to the shorter postoperative recovery period and hospital length of stay $[10,17]$. The duration of hospital stay in our study was relatively short, due to decreased pain and early removal of the chest tube (2.67 days vs. 4.15 days).

Limitations of the study are the low number of patients and its retrospective nature. A multicenter randomized controlled trial study with a larger sample size and a longer follow-up period would be a logical next step. It was attempted to keep interventional bias to a minimum by maintaining the same surgeons performing a constant approach, with the same routine, throughout the study.

\section{Conclusions}

This technique is a safe option for isolated benign rib lesions. It is more effective in patient recovery in the postoperative period and in the management of surgical pain. It is an advantageous alternative technique for experienced surgeons in selected patients.

\section{Conflict of interest}

The authors declare no conflict of interest.

\section{References}

1. Barrett NR. Primary tumors of rib. Br J Surg 1955; 43: 113-32.

2. Teitelbaum SL. Twenty years' experience with intrinsic tumors of the bony thorax at a large institution. J Thorac Cardiovasc Surg 1972; 63: 776-82.

3. Waller DA, Newman RJ. Primary bone tumours of the thoracic skeleton: an audit of the leeds regional bone tumour registry. Thorax 1990; 45: 850-5.

4. Shah AA, D’Amico TA. Primary chest wall tumors. J Am Coll Surg 2010; 210: 360-6. 
5. Smith SE, Keshavjee S. Primary chest wall tumors. Thorac Surg Clin 2010; 20: 495-507

6. Lukanich JM, Sugarbaker DJ. Chest wall and pleura. In: Sabiston Textbook of Surgery: The Biological Basis of Modern Surgical Practice. $17^{\text {th }}$ ed. Townsend CM, Beauchamp RD, Evers BM, Mattox KL (eds.). PA: Elsevier Saunders, Philadelphia 2004; 1715-7.

7. Incarbone M, Pastorino U. Surgical treatment of chest wall tumors. World J Surg 2001; 25: 218-30.

8. Ocakcioglu I, Alpay L, Demir M, et al. Is single port enough in minimally surgery for pneumothorax? Surg Endosc 2016; 30: 59-64.

9. Walker WS, Carnochan FM, Mattar S. Video-assisted thoracoscopic pneumonectomy. Br J Surg 1994; 81: 81-2.

10. Shanthanna $H$, Aboutouk D, Poon E, et al. A retrospective study of open thoracotomies versus thoracoscopic surgeries for persistent postthoracotomy pain. J Clin Anesth 2016; 35: 215-20.

11. Ohtsuka T, Wolf RK, Dunsker SB. Port-access first-rib resection. Surg Endosc 1999; 13: 940-2.

12. Nakagiri T, Akashi A, Shigemura N. Thoracoscopic rib resection using a Gigli saw. Ann Thorac Surg 2005; 80: 755-6.

13. Rocco G, Fazioli F, Martucci N, et al. Video-assisted thoracic surgery rib resection and reconstruction with titanium plate. Ann Thorac Surg 2011; 92: 744-5.

14. Cameron R. Robotic-assisted, minimally-invasive resection of high chest wall lesions. Presented: International Society for Minimally Invasive Cardiothoracic Surgery, Boston, MA, June 12, 2008. http://www.ismics.org/abstracts/2008/V10.html. Accessed: Feb 10, 2010.

15. Demmy TL, Nwogu CE, Yendamuri S. Thoracoscopic chest wall resection: what is its role? Ann Thorac Surg 2010; 89: 2142-5.

16. Bendixen M, Jorgensen OD, Kronborg, et al. Postoperative pain and quality of life after lobectomy via video-assisted thoracoscopic surgery or anterolateral thoracotomy for early stage lung cancer: a randomised controlled trial. Lancet Oncol 2016; 17: 836-44.

17. Hennon MW, Dexter EU, Huang M, et al. Does thoracoscopic surgery decrease the morbidity of combined lung and chest wall resection? Ann Thorac Surg 2015; 99: 1929-35.

Received: 20.03.2018, accepted: 10.06.2018. 